\title{
Machado-Joseph Disease
}

National Institute of Neurological Disorders and Stroke (NINDS)

\section{Source}

National Institute of Neurological Disorders and Stroke (NINDS). Machado-Joseph

Disease Information Page.

Machado-Joseph disease (MJD), which is also called spinocerebellar ataxia type 3, is a rare hereditary ataxia (ataxia is a medical term meaning lack of muscle control). The disease is characterized by slowly progressive clumsiness and weakness in the arms and legs, spasticity, a staggering lurching gait easily mistaken for drunkenness, difficulty with speech and swallowing, involuntary eye movements, double vision, and frequent urination. Some individuals also have dystonia (sustained muscle contractions that cause twisting of the body and limbs, repetitive movements, abnormal postures, and rigidity) or symptoms similar to those of Parkinson's disease. Others have twitching of the face or tongue, or peculiar bulging eyes. Almost all individuals with MJD experience vision problems, including double vision or blurred vision, loss of the ability to distinguish color and/or contrast, and inability to control eye movements. 